\title{
A comparative analysis of gallbladder torsion and acute gallbladder disease without torsion: a single-center retrospective case series study
}

\author{
Woo Young $\mathrm{Nho}^{1 \wedge}$, Jong Kun $\mathrm{Kim}^{2} \wedge$, Se Kook Kee ${ }^{3 \wedge}$ \\ ${ }^{1}$ Department of Emergency Medicine, CHA Gumi Medical Center, School of Medicine, CHA University, Gumi, Republic of Korea; ${ }^{2}$ Department of \\ Emergency Medicine, Kyungpook National University Chilgok Hospital, Kyungpook National University School of Medicine, Daegu, Republic of \\ Korea; ${ }^{3}$ Department of Surgery, CHA Gumi Medical Center, School of Medicine, CHA University, Gumi, Republic of Korea \\ Contributions: (I) Conception and design: WY Nho, SK Kee; (II) Administrative support: JK Kim; (III) Provision of study materials or patients: WY \\ Nho; (IV) Collection and assembly of data: WY Nho, JK Kim; (V) Data analysis and interpretation: SK Kee, WY Nho; (VI) Manuscript writing: All \\ authors; (VII) Final approval of manuscript: All authors. \\ Correspondence to: Se Kook Kee. Department of Surgery, CHA Gumi Medical Center, School of Medicine. CHA University, 12 Sinsi-ro 10 gil, Gumi \\ 39295, Republic of Korea. Email: kee39@cha.ac.kr.
}

Background: Acute gallbladder disease (AGD) is frequent in the emergency department (ED), and usually
requires surgical intervention. Gallbladder torsion (GT) is a rare entity among patients with AGD. There are
sparse reviews of GT's clinical characteristics, and there is no comparative study between them in the same
patient cohort. Therefore, we report the case series of GT, and compare the statistical differences between
GT and non-GT with AGD. Methods: We collected retrospective data from patients who visited ED with AGD and underwent emergency cholecystectomy between January 2005 and December 2020. We combined consecutive case series of GT and compared them with non-GT gallbladder disease.

Results: Six GT cases were diagnosed over the study duration. Five were female (83\%) and the average age was 77.8 years. All patients presented with abdominal pain, and the median duration of pain was two days. Only two cases were suspected for GT pre-operatively (33\%). One patient underwent laparotomy, and the others underwent laparoscopic cholecystectomy with a mean operation time of $59 \mathrm{~min}$. The torsion direction was of the same proportion in both directions; five (83\%) were rotated completely. The mean length of hospital stay was $9.3 \mathrm{~d}$ and outcomes were favorable in most GT cases. In the comparative study between GT and non-GT, age was higher in the GT group $(\mathrm{P}=0.048)$, and duration or severity of pain showed no statistical difference $(\mathrm{P}=0.528 ; \mathrm{P}=0.637$, respectively). Body temperature was higher in the non-GT group without statistical significance $(\mathrm{P}=0.074)$. Gallstones were present in $68.8 \%$ of the non-GT group, which is significantly higher than that of the GT group ( $\mathrm{P}=0.036)$.

Conclusions: Six exceptional GT cases were managed successfully. The overall characteristics of each GT case demonstrated similar with previous reviews. Our comparative analysis showed that age, pulse rate, serum creatinine level, and gallstone presence showed statistical differences. Contrary to the traditional knowledge of GT, some distinct features like sex, duration or severity of pain, and fever showed no significant differences within AGD in our research.

Keywords: Gallbladder torsion (GT); gallbladder volvulus; laparoscopic cholecystectomy; case series

Submitted May 10, 2021. Accepted for publication Aug 12, 2021.

doi: 10.21037/atm-21-2399

View this article at: https://dx.doi.org/10.21037/atm-21-2399

^ORCID: Woo Young Nho, 0000-0002-5956-9854; Jong Kun Kim, 0000-0002-0583-4368; Se Kook Kee, 0000-0001-7895-8604. 


\section{Introduction}

Acute gallbladder disease (AGD) is a common presentation of patients who visited the emergency department (ED) with upper abdominal pain (1). Approximately, $10-15 \%$ of the general population was estimated to have a gallstone, and one-third of patients with gallstone tend to develop acute cholecystitis (AC) (2). Furthermore, gallstone itself can generate colic pain. Gallbladder torsion (GT) is an unusual clinical entity, and hundreds of scattered cases were reported from the $19^{\text {th }}$ century (3). In contrast to another gallbladder (GB) disease, GT had been to know as frequent in elderly women, correlate lowly with gallstone, and usually revealing intraoperatively in those who undergo surgical management due to AGD. Only a few numbers of GT cases have been reported, however, they only described the characteristics and information within GT cases (4-6). A difference between GT and other GB diseases have been discussed in the past; however, these studies were neither comparative studies nor studies using statistical analysis (7). We report the case series of GT, and compare the statistical differences between GT and other GB diseases without torsion in a single center. We present the following article in accordance with the PROCESS reporting checklist (available at https://dx.doi.org/10.21037/atm-21-2399) (8).

\section{Methods}

\section{Study design}

CHA Gumi Medical Center is a secondary referral center in South Korea. Annually, more than 35,000 patients visited our Level 1 regional ED and the institution's surgical volume exceeds 8,000 cases every year. We retrospectively collected medical records of patients who visited ED with GB disease, and managed surgically from January 2005 to December 2020. A total of 3,737 individual patients have undergone an emergency cholecystectomy with AGD in our hospital, and we identified six cases of GT from those of 3,737 patients $(0.16 \%)$. There was a paninstitutional systemic upgrade to the electronic medical system in early 2010. Unfortunately, large numbers of clinical data in the early stages of our study period were missing during the system migration. Therefore, we planned our comparison study with the data from January 2014, when the second GT case of GT occurred, to December 2020. Two thousand twenty-five individuals underwent emergency cholecystectomy due to AGD during that period. From the second GT case, every GT surgery was performed by laparoscopy. We excluded 46 patients per the exclusion criteria: (I) incomplete demographic/ clinical data, (II) conversion from laparoscopic approach to open cholecystectomy. Finally, we included data of 1,979 individual patients with AGD including five GT cases and completed a comparative analysis (Figure 1).

\section{Data collection and variables}

We collected demographic and clinical data retrospectively, including patient age, sex, vital signs, physical examination, and the duration of symptoms and pain severity on ED arrival was collected from the medical records. The severity of pain was measured by the numeric rating scale (NRS), a simple tool widely used in the ED (9). The patients rated their pain from 0 to 10 , which a score of 0 indicates "no pain at all" and a score of 10 represents "worst imaginable pain, the most intense pain imaginable" (9). The patients underwent evaluations, including laboratory tests and radiological studies of ultrasonography (USG) or computed tomography (CT), to determine the AGD in the ED preoperatively. Each study's results, which were associated with the diagnosis of $\mathrm{AC}$ or important factors for grading the severity of AC, were obtained (10). We collected general surgical data, like the time from ED arrival to surgery, operation duration, direction and degree of GT, presence of gallstones, pathology reports from operation records. We also reviewed the length of hospital stay after surgery and the presence of postoperative complications or re-admission after discharge were reviewed from medical records.

\section{Statistical analysis}

Data were analyzed using IBM SPSS Statistics v. 25 (IBM Corp., Armonk, NY, USA). Categorical variables were presented as frequencies and percentages, whereas continuous variables were reported as means \pm standard deviations (SD) or as medians and interquartile ranges (IQRs, 25th-75th percentile). The chi-square or Fisher's exact test was used to compare categorical variables. A $t$-test or one-way analysis of variance and Mann-Whitney U-test were used to compare continuous variables. All tests were two-tailed, and a $\mathrm{P}$ value of $<0.05$ was considered to indicate statistically significant.

\section{Ethics statement}

The study was conducted in accordance with the 


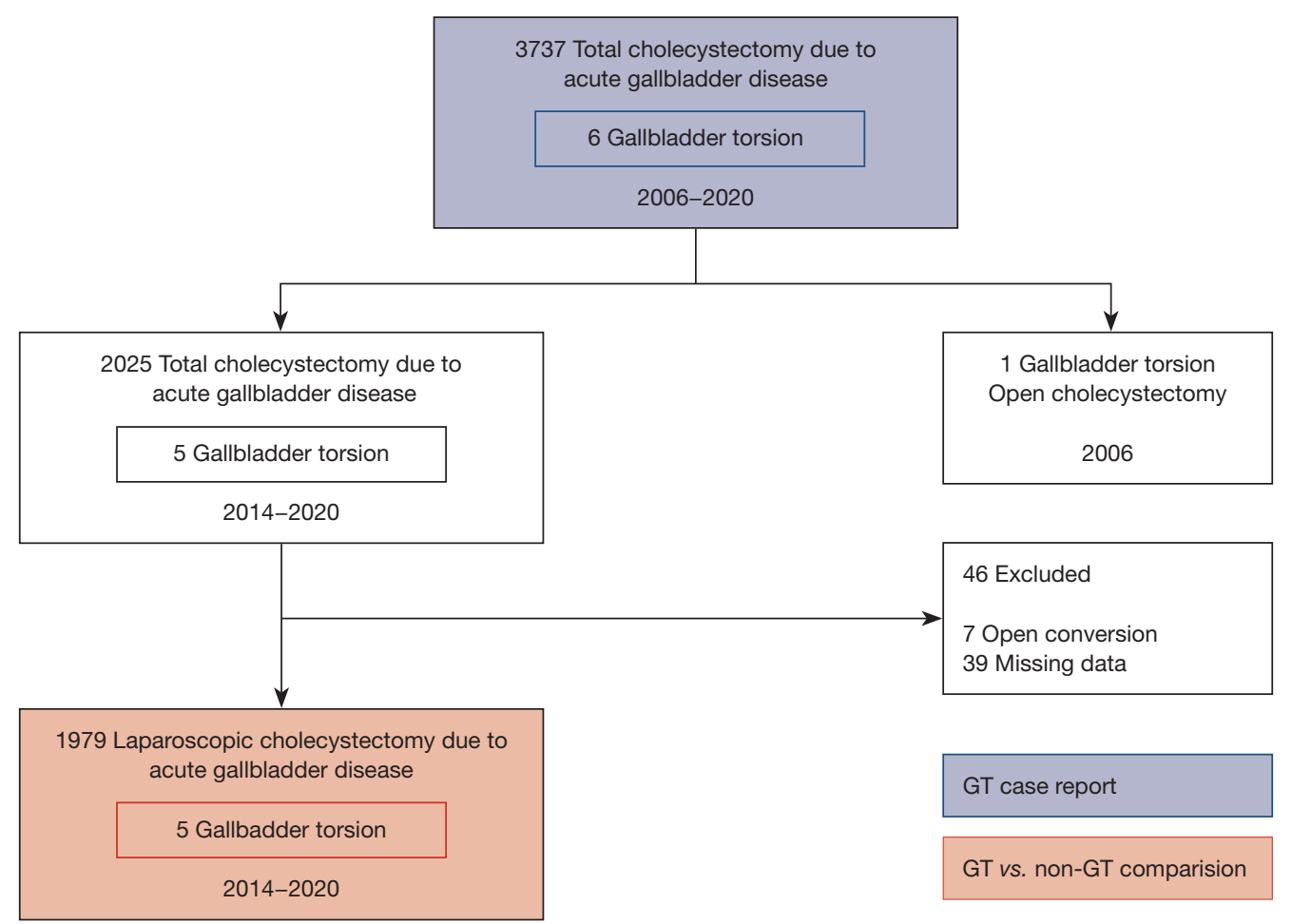

Figure 1 Flowchart of the study. Blue box indicates a case-series report of gallbladder torsion. Red box indicates a comparative analysis between gallbladder torsion and acute gallbladder disease without torsion.

Declaration of Helsinki (as revised in 2013). The study was approved by institutional ethics review board of CHA Gumi University Hospital (approval number: GM21-04) and individual consent for this retrospective analysis was waived.

\section{Results}

\section{Case series report of $G T$}

Over the study duration, six individual cases were diagnosed for GT. Detailed information about each GT case including general demographic characteristics and clinical data are summarized in Table 1. Five were female $(83 \%)$ and the age range was 69-90 years, with an average of 77.8 years. There was no pediatric case. All six patients represented upper abdominal area pain upon ED arrival, three of the epigastric area $(50 \%)$ and another three of the right upper quadrant area $(50 \%)$. The median duration of pain was two days (ranging from 1 to 3). Nausea was most frequent associating symptoms $(\mathrm{n}=4,67 \%)$ and other symptoms were vomiting $(n=2,33 \%)$, anorexia $(n=1,17 \%)$, and chill $(n=1$, $17 \%)$. There were no hypotension, tachycardia, and fever for all patients. Four patients (67\%) showed leukocytosis in the laboratory studies, and neutrophilia was observed in five patients ( $83 \%$ ). Two cases (33\%) had highly elevated levels of erythrocyte sedimentation rate (ESR) and C-reactive protein (CRP). All patients had a radiological study of CT scans and three of them had a USG for preoperative evaluation. GT was suspected preoperatively in two cases (33\%), each from CT and USG (Figure 2). In case No. 2, a whirl sign of cystic artery was observed in CT scan (Figure 2B). USG findings of distended GB without vascular flow, and irregular GB wall thickening were reported in case No. 5 (Figure 2E). The first patient (No. 1) underwent open cholecystectomy with a midline incision, and others underwent laparoscopic cholecystectomy (LC). The mean time from ED arrivals to begin of operation was $9.8 \mathrm{~h}$ (from 3.5 to $27.6 \mathrm{~h}$ ) and the mean operation time was $59 \mathrm{~min}$ (from 35 to $120 \mathrm{~min}$ ). Three of six cases $(50 \%)$ were identified that twisted in a counter-clockwise direction, and another half were in a clockwise pattern. Most ( $\mathrm{n}=5,83 \%)$ were completely rotated. In one case (No. 6), torsion was identified at the first view of the laparoscope by the surgeon, however, spontaneous detorsion was processed during the surgery. For that reason, this specific case was considered incomplete torsion. GT in 


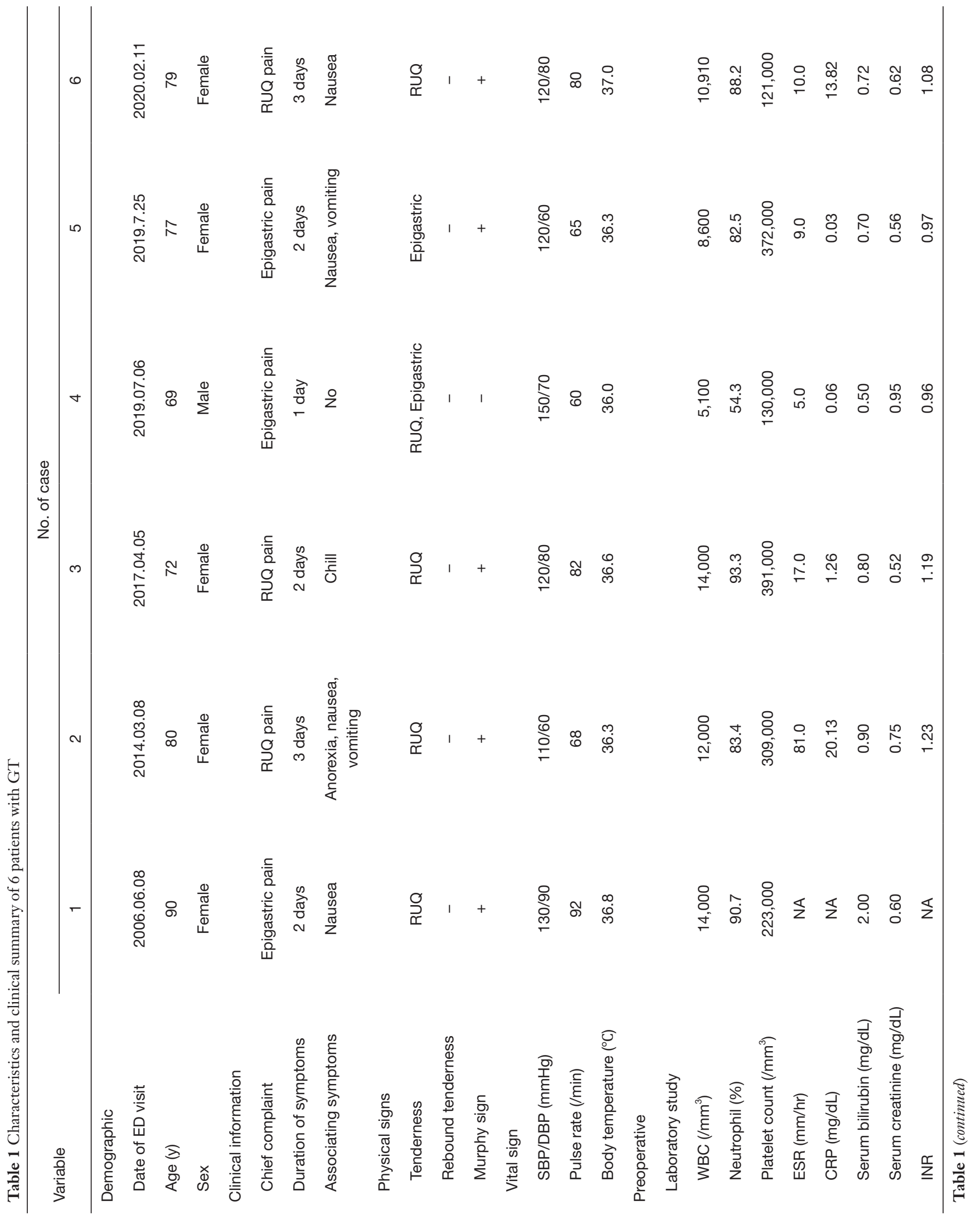




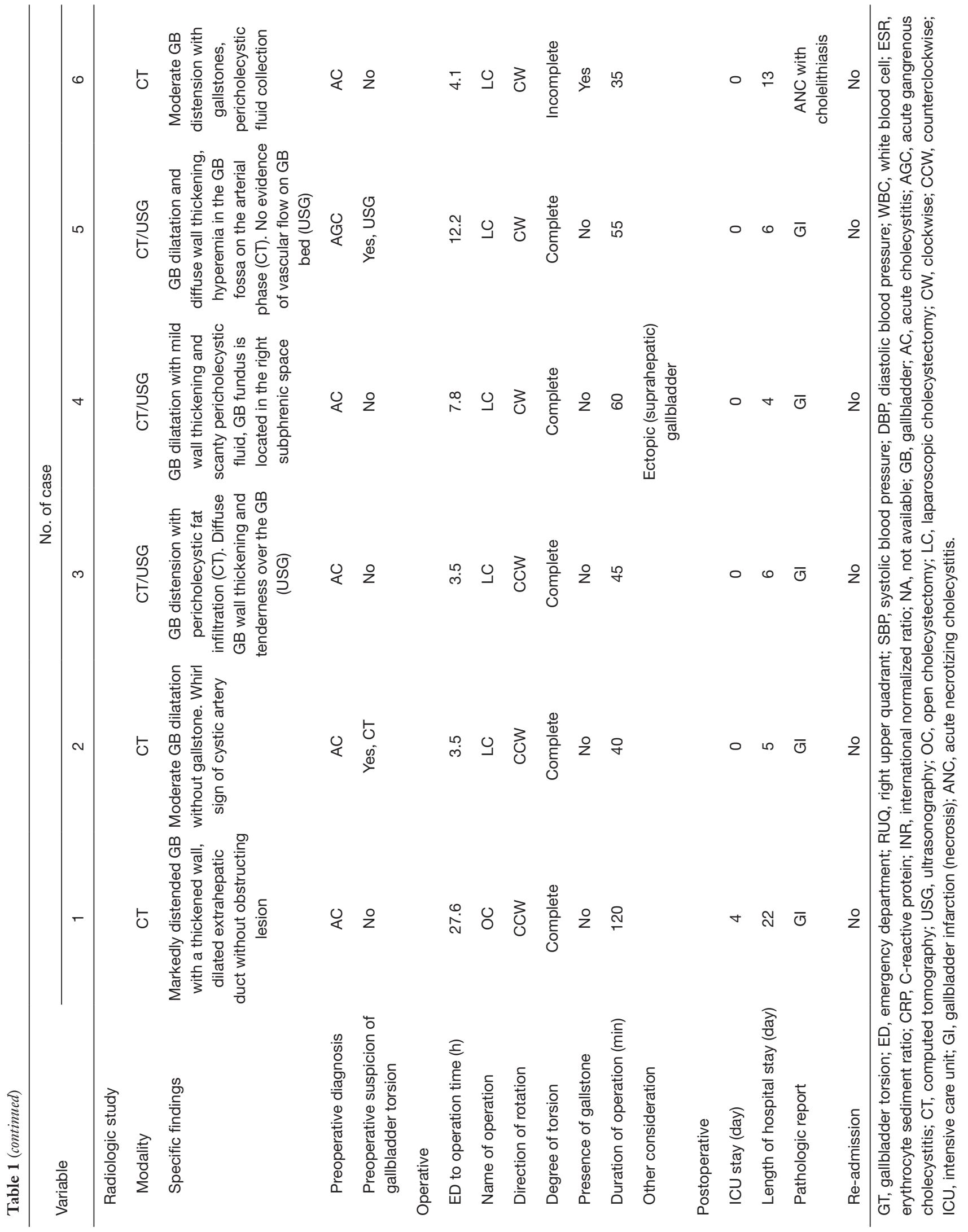



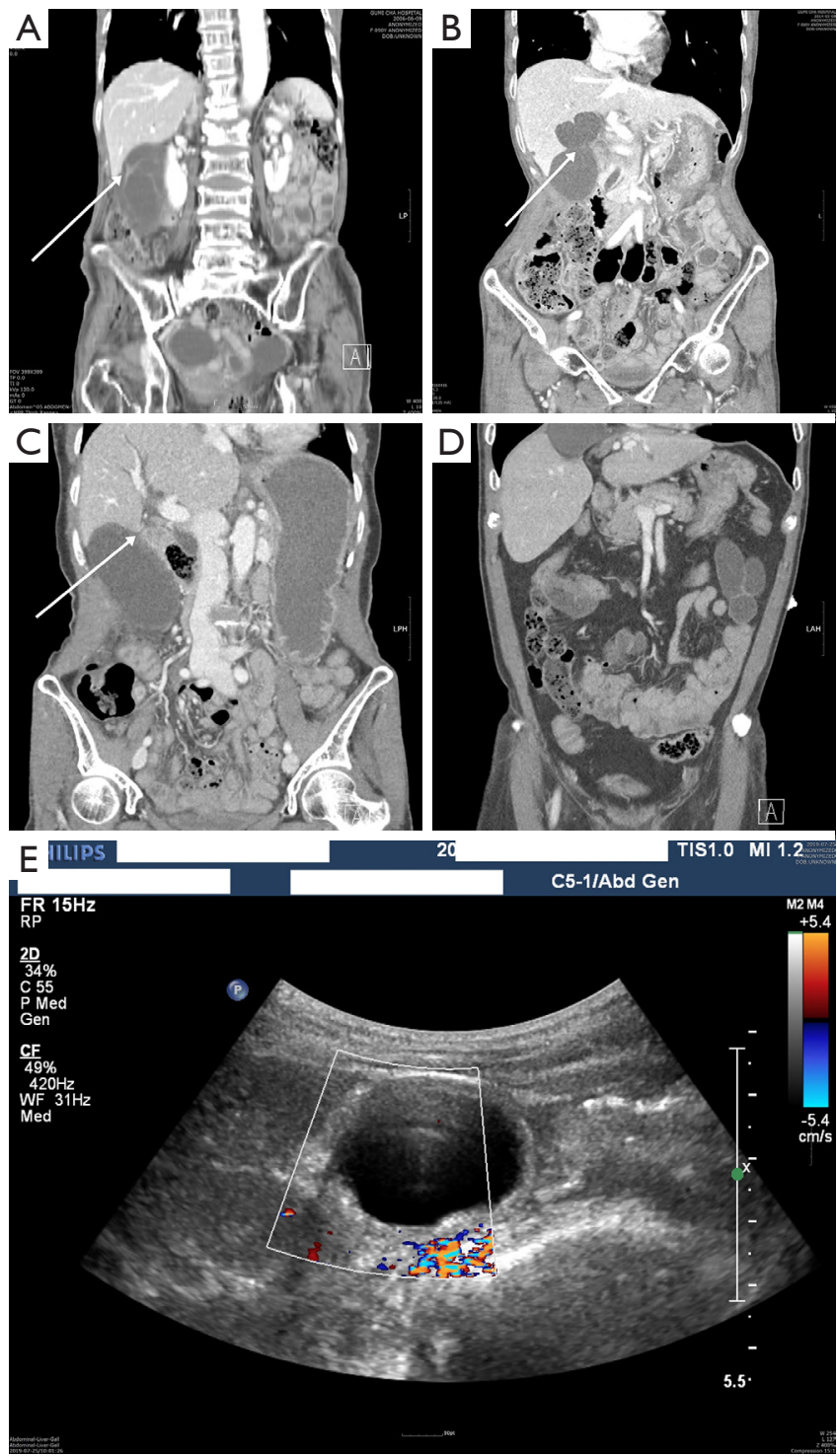

Figure 2 Radiologic study. (A) Case No. 1, computed tomography, dilated gallbladder with a diffusely thickened gallbladder wall and abruptly narrowed neck (white arrow), retrograde review after cholecystectomy. (B) Case No. 2, computed tomography, distended gallbladder with whirl sign (white arrow) suspected preoperatively. (C) Case No. 3, Computed tomography, distended gallbladder with beak sign (white arrow), retrograde review after cholecystectomy. (D) Case No. 4, computed tomography, the suprahepatic gallbladder. (E) Case No. 5, Doppler image, no vascular flow on gallbladder bed and suspected preoperatively.

the suprahepatic region was observed in case No. 4 (Figure 3). In the pathological report, five cases $(83 \%)$ were diagnosed with gallbladder infarction (necrosis), and one case (17\%) had gallstones (Figure 4). Only one patient was admitted to the intensive care unit (ICU) after surgery and stayed for four days. The mean length of hospital stay after surgery was $9.3 \mathrm{~d}$ (from 4 to $22 \mathrm{~d}$ ). None of the patients were reported with postoperative complications, and there was no re-admission after discharge.

\section{Comparison of GT and non-GT gallbladder disease}

The retrospective data analysis was performed from January 2014 to December 2020. A total of 1,979 patients, who had LC with AGD were included. Table 2 compares parameters, such as primary demographic data and clinical information, including preoperative evaluations, operative interventions, and postoperative factors between GT and non-GT groups. The age was significantly higher in the GT group ( $\mathrm{P}=0.048)$. In contrast to the GT group, the sex of the non-GT group showed a similar ratio in both males and females. The duration of pain and NRS score showed no statistical difference in both groups $(\mathrm{P}=0.528 ; \mathrm{P}=0.637$, respectively). The pulse rate was faster in the non-GT group $(\mathrm{P}=0.032)$, and body temperature was mildly higher in the non-GT group; however, there was no statistical significance $(\mathrm{P}=0.074)$. The serum creatinine level was higher in the non-GT group with statistical significance $(\mathrm{P}=0.043)$. The time from ED arrival to begin of operation, and the duration of operation were longer in the nonGT group, however, the difference was not statistically significant $(\mathrm{P}=0.087 ; \mathrm{P}=0.942$, respectively). In the non-GT group, about two-thirds of patients had gallstones (68.8\%), and this is a significantly higher proportion than that of the GT group $(\mathrm{P}=0.036)$.

\section{Discussion}

To the best of our knowledge, this is the first study on the statistical comparison between GT and non-GT GB disease, additionally with an exceptional case series report of the GT. There was no statistical inference of GT for more than a century. Only a few individual researchers have described their opinions on GT compared with other GB disease, however they documented without scientific evidence (7). Furthermore, mean time from ED to surgery of $15.3 \mathrm{~h}$ for the emergency cholecystectomy, which has been taken far shorter than guideline recommended (within 7 days from hospital visit) and other research, and this properly reflect the enthusiasm of our surgical team to the patients with AGD $(1,2)$. This is the novelty and unique 

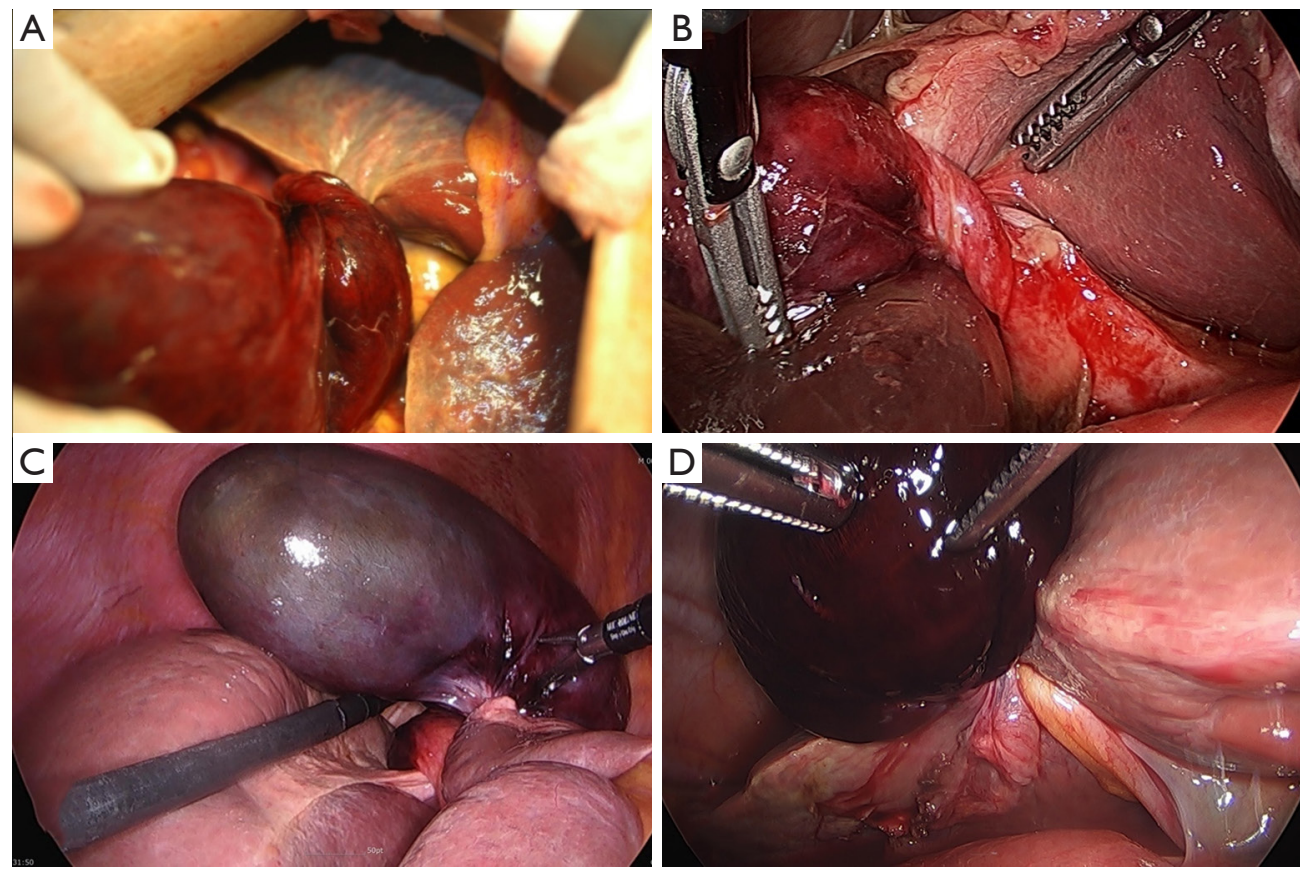

Figure 3 Operative findings. (A) Case No. 1, counter-clockwise torsion, laparotomy. (B) Case No. 2, counter-clockwise torsion, laparoscopic cholecystectomy. (C) Case No. 4, clockwise torsion, laparoscopic cholecystectomy. (D) Case No. 5, clockwise torsion, laparoscopic cholecystectomy.
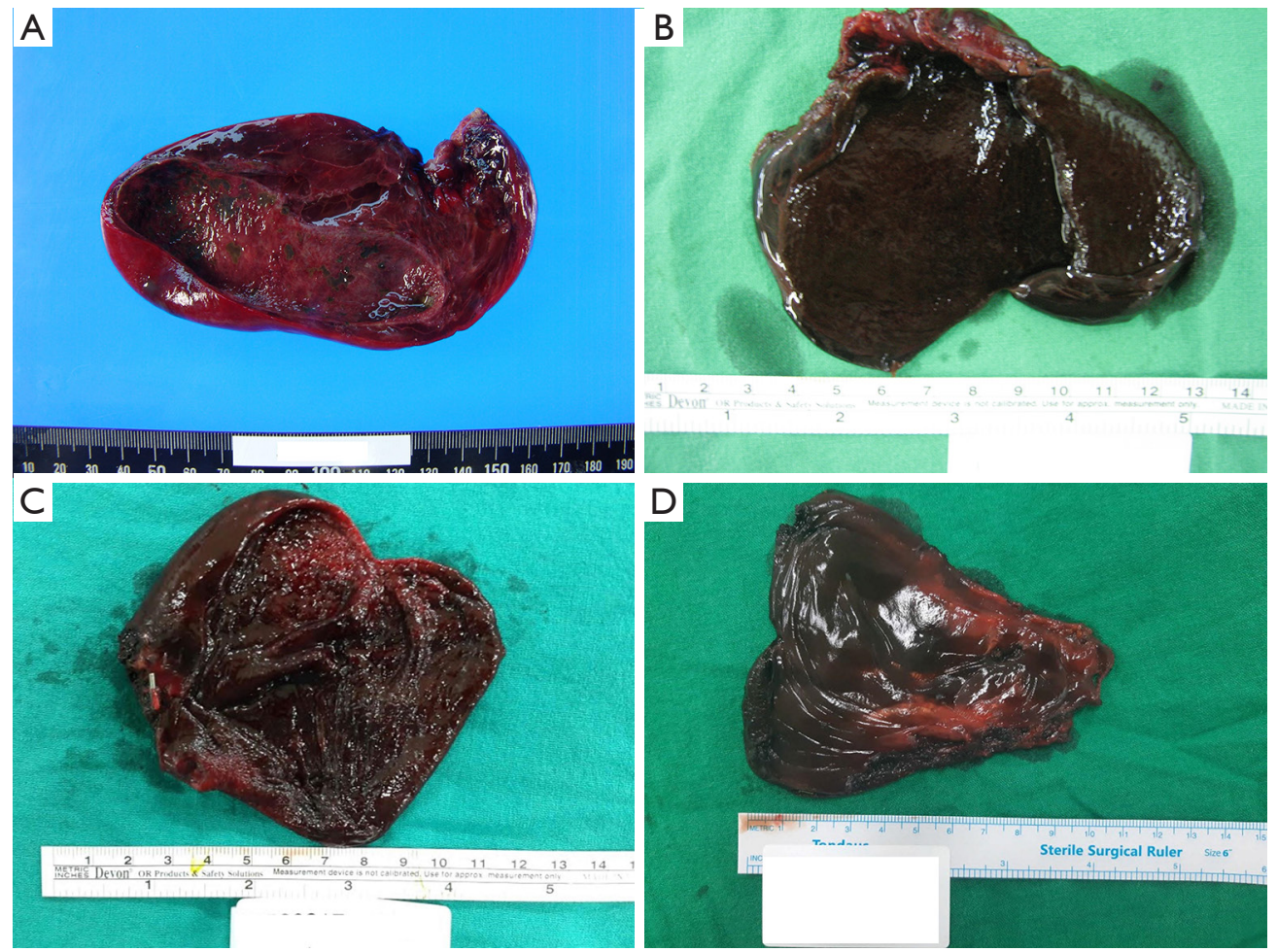

Figure 4 Resected gallbladder. (A) Case No. 1, gallbladder infarction (necrosis). (B) Case No. 2, gallbladder infarction (necrosis). (C) Case No. 3, gallbladder infarction (necrosis). (D) Case No. 4, gallbladder infarction (necrosis). 
Table 2 Comparison between GT group and non-GT group

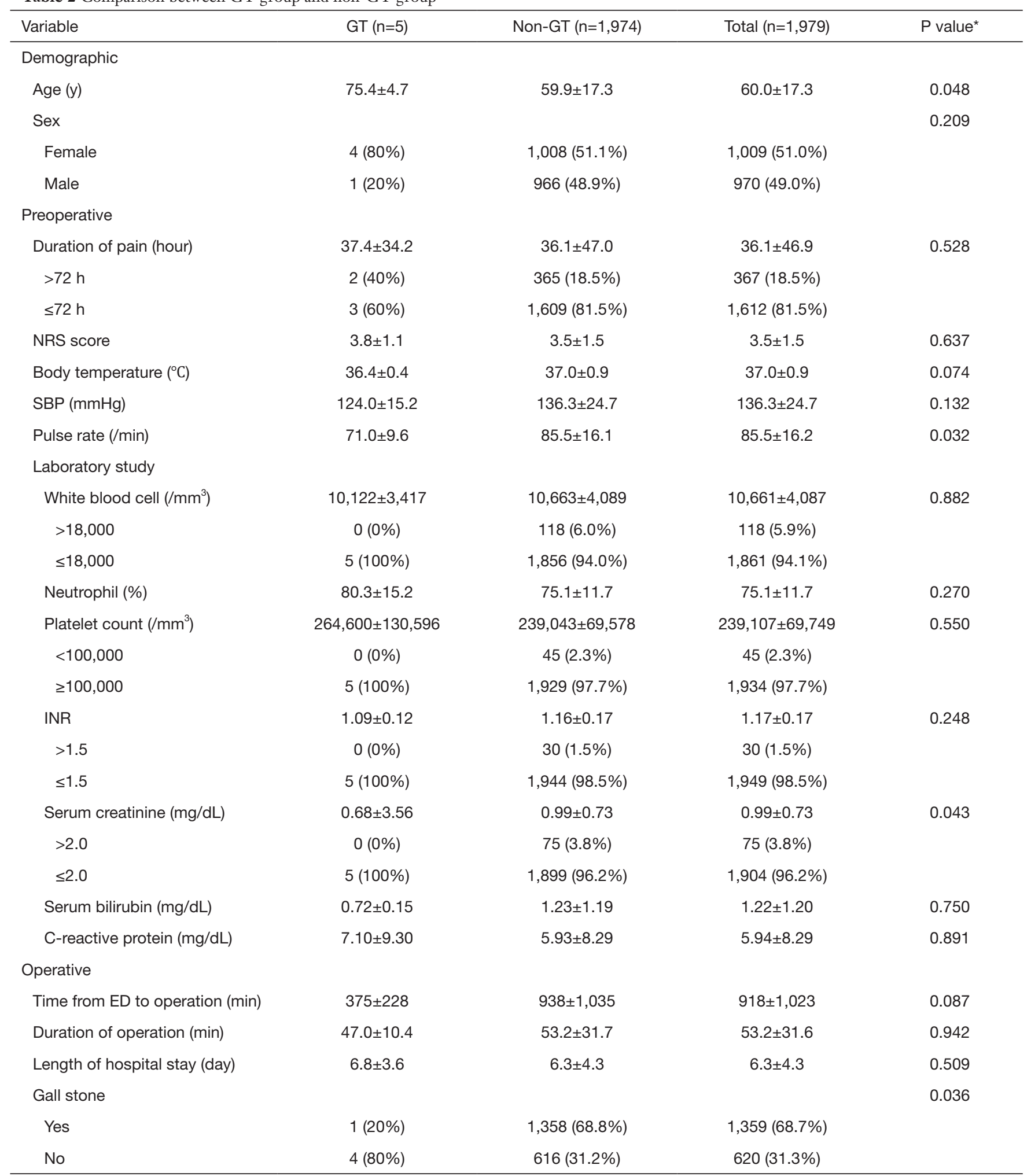

Values are mean $\pm \mathrm{SD}$ or number (\%). ${ }^{*}, \mathrm{P}$ values reflect Mann-Whitney $\mathrm{U}$ tests and Fischer exact chi-square comparisons where appropriate. GT, gallbladder torsion; NRS, numeric rating scale; SBP, systolic blood pressure; INR, international normalized ratio; ED, emergency department; SD, standard deviation. 
aspect of our study, despite the small numbers of GT could be related to the low statistical power.

The term "gallbladder torsion" or "gallbladder volvulus" was widely used in the presentation of twisted GB. When the GB rotated more than $180^{\circ}$, this can be classified as complete torsion. Otherwise, incomplete torsion is defined as the gallbladder rotated less than $180^{\circ}(11)$. As torsion becomes complete, the GB's blood supply is directly affected, causing infarction and necrosis (12). In our study, $83 \%(n=5)$ of GT was complete and this would be comparable to the previous systematic review, which reports the $82 \%$ of GT were complete (11). The etiology of GT is not fully known. Association with anatomical anomalies of the long cystic pedicle is a widely accepted theory. Four to five percent of the general population has reportedly to have a congenital anomaly and they are more vulnerable to torsion $(13,14)$. The term "floating gallbladder" is related to long cystic duct and the position of GB is free from the liver bed (3). Another important factor is the loss of visceral fat in old age, the so-called "visceroptosis". As the result of falling or dropping of the stomach or intestine, more anatomical space could be reserved, and contribute to the GB mobility $(7,12,15)$. Anatomical variation of other organs can promote GT, like case No. 4 in our report, which was related to the atrophy of the liver segment 4 (16). In contrast to many cases demonstrated a twisted cystic pedicle, torsion of GB body had been reported, rarely (17). The direction of GB torsion is related to the affected organ. The gastric hyperperistalsis causes clockwise rotation, and counterclockwise torsion has been affected by the colon $(11,12)$. In our research, GT consisted of the same proportion in both directions (3:3 ratio), comparable with a previous systematic review (11).

Traditionally, GT is known as associated with old-aged females $(11,18)$. GT has been reported as preponderance in females, 3-5 times more than males and the mean and median age were 64 and 77 , respectively $(6,11,19)$. Our research demonstrated a 5:1 sex ratio of GT, in contrast to the non-GT group that showed a similar proportion of both sexes. The age of the GT group revealed significantly older than the non-GT group in this analysis $(\mathrm{P}=0.048)$. Approximately $30 \%$ of GT patients presented gallstones in previous reports $(7,11,20)$. In this study, only one of six GT cases $(17 \%)$ demonstrated a gallstone. However, more than two-thirds of patients in the non-GT group presented with gallstone, and there was a significant difference between both groups. The symptoms of GT are similar to other GB diseases, and it is difficult to make a differential diagnosis only with their symptoms. In this study, all GT patients demonstrated upper abdominal pain, and symptoms of nausea/vomiting were most frequently associated, which is similar to the previous GT report (6,7). Additionally, Nakao et al. proposed that the GT had their clinical characteristics, distinct from AC: acute onset of pain, infrequent fever, jaundice, and low prevalence of gallstones (7). Here, the presence of gallstones was shown to be significantly higher in the non-GT group. However, there was no significant difference in the duration of pain or body temperature between GT and other GB diseases without torsion in our analysis. Additionally, the severity of pain did not show statistical differences between GT and non-GT groups, either.

Most GT has been diagnosed during operation. Various radiologic studies have played an essential role in suspicion and diagnosing GT, and around 10-26\% were diagnosed before surgical intervention $(7,11,14,20,21)$. The cystic duct knot sign, floating gallbladder are helpful USG signs, and torsion could be suspected by the restricted vascular flow on color Doppler $(13,22)$. Among our case series, only one GT case was suspected by USG preoperatively. The "whirl and beak sign" accompanying abnormal position of distended GB are typical findings of CT scans in the GT $(19,20,23,24)$. Other imaging modalities of magnetic resonance cholangiopancreatography (MRCP), hepatobiliary iminodiacetic acid (HIDA) scans, and upper GI endoscopy offer a diagnostic value (2). A laboratory study has been routinely evaluated for patients with abdominal pain in ED. Even though various blood tests offer clinical value in biliary disease, GT itself does not correlate with tests. According to Tokyo Guidelines, some blood test has diagnostic value and perspective factors for grading the severity of AC (10). An elevated level of white blood cell count and CRP consists of criteria for diagnosing AC. The serum creatinine level, international normalized ratio (INR), and platelet count are related to the severity grading for AC. Another guideline proposed that neutrophil count was independently associated with the diagnosis of AC (2). Once the GB twists, concomitant necrosis follows, and finally, it causes inflammation. Therefore, these laboratory results may play a role as predictors of the progress and severity of GT. Most GT cases in our study did not meet the criteria for Grade II or III AC. Furthermore, there was no statistical difference between the GT and non-GT groups for most of the laboratory values except serum creatinine level $(\mathrm{P}=0.043)$.

Laparoscopy is the standard method of choice for 
treatment, regardless of a pre-operative diagnosis was made or not. As the characteristics of low pre-operative diagnostic rate, surgery has both diagnostic and therapeutic value in GT. LC is recommended as the first-line surgical treatment of acute inflammatory GB disease (2). In addition, the GT should be managed promptly by the surgical method after diagnosis $(18,24)$. In recent reports, torsion was managed successfully by laparoscopy $(5,7,25)$ and some surgeons have tried the single incision laparoscopic method (26). In our study, the laparoscopic approach was successful in total GT cases. A surgical profile like the duration of operation or length of hospital stay showed no statistical difference between GT and non-GT groups. The time from ED arrival to begin of operation showed longer in the non-GT group, and this result was considered that alternative option like percutaneous drainage or medical treatment was chosen before surgery. The mortality of GT has been reported as $5-6 \%(7,11)$. Fortunately, out studies had no fatal case, despite the gangrenous change of several cases already confirmed.

There were several limitations in our study. As mentioned above, due to their rarity, a few GT could be related to the low statistical power of our analysis. Statistical difference in pulse rate and serum creatinine level needs more discussion. A further large-scale investigation should be considered to evaluate the risk factor or predictable factor of GT. Some cases, which were excluded from comparison due to open conversion, may lead to selection bias. Our study mainly analyzed GT from other GB diseases without torsion. Five cases of GT, which included for comparative study, were managed laparoscopically, and baseline characteristics of a candidate in both groups should be equalized for their comparative analysis. Open conversion has been recommended to $\mathrm{AC}$ with complications like severe local inflammation, adhesion, bleeding, or biliary injury (2). As a result, the case of AC with severe grades could be excluded from the analysis.

\section{Conclusions}

We report the exceptional case series of six with GB torsion without unfavorable outcomes. Overall characteristics of each case of GT showed a comparable result with previous reports. As a result of the comparative analysis between GT and other GB diseases without torsion, we found age, pulse rate, serum creatinine level, and presence of gallstone revealed statistical differences. Contrary to the traditional knowledge about GT, some distinct features like sex, duration or severity of pain, and fever showed no significant differences within AGD in our research.

\section{Acknowledgments}

Funding: None.

\section{Footnote}

Reporting Checklist: The authors have completed the PROCESS reporting checklist. Available at https://dx.doi. org/10.21037/atm-21-2399

Data Sharing Statement: Available at https://dx.doi. org/10.21037/atm-21-2399

Conflicts of Interest: All authors have completed the ICMJE uniform disclosure form (available at https://dx.doi. org/10.21037/atm-21-2399). The authors have no conflicts of interest to declare.

Ethical Statement: The authors are accountable for all aspects of the work in ensuring that questions related to the accuracy or integrity of any part of the work are appropriately investigated and resolved. The study was conducted in accordance with the Declaration of Helsinki (as revised in 2013). The study was approved by institutional ethics review board of CHA Gumi University Hospital (Approval number: GM21-04) and individual consent for this retrospective analysis was waived.

Open Access Statement: This is an Open Access article distributed in accordance with the Creative Commons Attribution-NonCommercial-NoDerivs 4.0 International License (CC BY-NC-ND 4.0), which permits the noncommercial replication and distribution of the article with the strict proviso that no changes or edits are made and the original work is properly cited (including links to both the formal publication through the relevant DOI and the license). See: https://creativecommons.org/licenses/by-nc-nd/4.0/.

\section{References}

1. Cox DRA, Fong J, Liew CH, et al. Emergency presentations of acute biliary pain: changing patterns of management in a tertiary institute. ANZ J Surg 2018;88:1337-42.

2. Pisano M, Allievi N, Gurusamy K, et al. 2020 World 
Society of Emergency Surgery updated guidelines for the diagnosis and treatment of acute calculus cholecystitis. World J Emerg Surg 2020;15:61.

3. Wendel AV. VI. A Case of Floating Gall-Bladder and Kidney complicated by Cholelithiasis, with Perforation of the Gall-Bladder. Ann Surg 1898;27:199-202.

4. Ikematsu Y, Yamanouchi K, Nishiwaki Y, et al. Gallbladder volvulus: experience of six consecutive cases at an institute. J Hepatobiliary Pancreat Surg 2000;7:606-9.

5. Ezaki S, Tomimaru Y, Noguchi K, et al. Surgical Results of Laparoscopic Cholecystectomy for Gallbladder Torsion. Am Surg 2019;85:471-3.

6. Moser L, Joliat GR, Tabrizian P, et al. Gallbladder volvulus. Hepatobiliary Surg Nutr 2021;10:249-53.

7. Nakao A, Matsuda T, Funabiki S, et al. Gallbladder torsion: case report and review of 245 cases reported in the Japanese literature. J Hepatobiliary Pancreat Surg 1999;6:418-21.

8. Agha RA, Sohrabi C, Mathew G, et al. The PROCESS 2020 Guideline: Updating Consensus Preferred Reporting Of CasESeries in Surgery (PROCESS) Guidelines. Int J Surg 2020;84:231-5.

9. Karcioglu O, Topacoglu H, Dikme O, et al. A systematic review of the pain scales in adults: Which to use? Am J Emerg Med 2018;36:707-14.

10. Yokoe M, Hata J, Takada T, et al. Tokyo Guidelines 2018: diagnostic criteria and severity grading of acute cholecystitis (with videos). J Hepatobiliary Pancreat Sci 2018;25:41-54.

11. Reilly DJ, Kalogeropoulos G, Thiruchelvam D. Torsion of the gallbladder: a systematic review. HPB (Oxford) 2012;14:669-72.

12. Hwang Y, Kulendran K, Ashworth J. Expect the Unexpected: Torsion of the Gallbladder, a Rare Cause for Acute Cholecystitis. Cureus 2018;10:e3726.

13. Ohkura Y, Hashimoto M, Sasaki K, et al. Complete acute gallbladder torsion diagnosed with abdominal ultrasonography and colour Doppler imaging. BMJ Case Rep 2013;2013:bcr2012008460.

14. Boer J, Boerma D, de Vries Reilingh TS. A gallbladder

Cite this article as: Nho WY, Kim JK, Kee SK. A comparative analysis of gallbladder torsion and acute gallbladder disease without torsion: a single-center retrospective case series study. Ann Transl Med 2021;9(18):1399. doi: 10.21037/atm-21-2399 torsion presenting as acute cholecystitis in an elderly woman: A case report. J Med Case Rep 2011;5:588.

15. McHenry CR, Byrne MP. Gallbladder volvulus in the elderly. An emergent surgical disease. J Am Geriatr Soc 1986;34:137-9.

16. Nho WY, Kim JO, Nam SY, et al. Torsion of suprahepatic gall bladder. J Minim Access Surg 2021;17:382-4.

17. Kwon HJ, Kim SG. A rare case of gallbladder torsion along the axis of body: a case report. Korean J Hepatobiliary Pancreat Surg 2015;19:82-5.

18. Khandelwal C. Gallbladder disease in an elderly patient. JAMA Surg 2013;148:891-2.

19. Kitagawa H, Nakada K, Enami T, et al. Two cases of torsion of the gallbladder diagnosed preoperatively. J Pediatr Surg 1997;32:1567-9.

20. Layton B, Rudralingam V, Lamb R. Gallbladder volvulus: it's a small whirl. BJR Case Rep 2016;2:20150360.

21. Gupta V, Singh V, Sewkani A, et al. Torsion of gall bladder, a rare entity: a case report and review article. Cases J 2009;2:193.

22. Dasyam AK, Koo J, Stahlfeld Miller M, et al. The cystic duct knot sign: case report with description of a new ultrasound sign of gallbladder torsion. Emerg Radiol 2015;22:445-7.

23. Tajima Y, Tsuneoka N, Kuroki T, et al. Clinical images. Gallbladder torsion showing a "whirl sign" on a multidetector computed tomography scan. Am J Surg 2009;197:e9-10.

24. Younan G, Schumm M, Ali F, et al. Gallbladder Volvulus in a Patient with Type I Choledochal Cyst: A Case Report and Review of the Literature. Case Rep Surg 2016;2016:5626531.

25. Nguyen T, Geraci A, Bauer JJ. Laparoscopic cholecystectomy for gallbladder volvulus. Surg Endosc 1995;9:519-21.

26. Matsubara K, Urushihara T, Oshita A, et al. Singleincision laparoscopic cholecystectomy for gallbladder torsion: A case report and literature review. Asian J Endosc Surg 2018;11:165-8. 